\title{
ÉTICA DE LA PRESCRIPCIÓN MÉDICA EN UN PAÍS PAUPERIZADO
}

\author{
Jorge Luis Manzini*
}

Resumen: Este artículo hace una sucinta revisión de los aspectos éticos de la prescripción a nivel "micro", el de la relación terapéutica, con fundamento en la "teoría de los principios" (de Georgetown), y en los aspectos técnicos de la correcta prescripción según la lex artis, sustentada en la denominada "medicina basada en la evidencia" y en las recomendaciones y publicaciones de la OMS.

Luego, se aborda el tema de la prescripción médica en tanto problema de salud pública. Se comenta su impacto social y las presiones indebidas que sufren los médicos para prescribir, especialmente por parte de la industria farmacéutica.

Se provee a continuación un marco jurídico, centrado en el llamado Derecho Internacional de los Derechos Humanos. Se considera que podría lograrse el acceso universal a los medicamentos, entendidos como bien social, de fundamental importancia para efectivizar el Derecho a la Asistencia Sanitaria. Se revisan las distintas justificaciones éticas que sostienen este derecho, especialmente la teoría de la justicia de John Rawls.

Palabras clave: bioética, salud pública, prescripción, industria farmacéutica

\section{ETHICAL MEDICAL PRESCRIPTION IN A VERY POOR COUNTRY}

Abstract: This paper pretends to draw a brief review upon the ethical aspects of a prescription at a "micro" level, that is of the therapeutic relationship based on the Georgetown "theory of principles" and on the technical aspects of a correct prescription according to the lex artis, sustained on the so-called "evidence based medicine" and on the WHO's requests and publications.

It then turns to the subject of the medical prescription as a problem of Public Health. It stops at its social impact and at the undue pressures that doctors must suffer in order to prescribe, especially those coming from the pharmaceutical industry.

It also offers a juridical framework focused on the Human Rights' International Law. Universal access to medication should be reached -understood as a social good of fundamental importance- in order to attain the Right to Sanitary Assistance. Several ethical justifications that hold up this right are taken into account, especially Rawls' theory of justice

Key words: bioethics, public health, prescription, pharmaceutical industry

\section{A ÉTICA DA PRESCRIÇÃO MÉDICA NUM PAÍS PAUPERIZADO}

Resumo: Este texto se inicia como uma breve revisão dos aspectos éticos da prescrição em nível "micro", ou seja, a da relação terapêutica, com fundamento na "teoria dos princípios (Georgetown)", e dos aspectos técnicos da correta prescrição segundo a lex artis, sustentada na denominada "medicina baseada em evidência", as recomendações e publicações da OMS.

A seguir se aborda o tema da prescrição médica, enquanto problema de saúde pública. Comenta seu impacto social e as pressões indevidas que sofrem os médicos para prescrever, especialmente da indústria farmacêutica.

Continuando, apresenta um marco jurídico, centrado no chamado Direito Internacional dos Direitos Humanos. Considerase que se poderia conseguir o acesso universal aos medicamentos, entendidos como um bem social, de fundamental importância para efetivar o Direito à Assistência Sanitária. Revisam-se as diferentes justificações éticas que sustentam este direito, especialmente a teoria da justiça de Rawls.

Palavras chave: bioética; saúde pública; prescrição; indústria farmacêutica

Programa de Bioética de la Universidad Nacional de Mar del Plata, Argentina

Correspondencia: manzmdq@sinectis.com.ar 
Mi propósito es presentarles una discusión acerca de la prescripción médica en tanto problema de salud pública, problema que corresponde a la dimensión macro de la bioética (la bioética de poblaciones). De todos modos, trataré de dar también alguna idea de la dimensión micro del problema, la receta con que el médico suele terminar una entrevista con su paciente individual.

Como marco teórico me parecen apropiados tanto la llamada "Teoría de los principios", de Georgetown, EE.UU.(1), como el enfoque desde el Derecho Internacional de los Derechos Humanos, con la consideración del Derecho a la Salud como un derecho humano fundamental. La primera, en cuanto a la interacción del médico con el paciente en su consultorio, y el segundo especialmente en lo que se refiere a la dimensión social de la prescripción médica.

En el ámbito de la entrevista médica, el médico y el paciente están solos "como en una isla desierta", decía Schweninger a fines del siglo XIX en el Berlín de Bismarck ${ }^{1}$. En este contexto, de acuerdo a la teoría "de Georgetown"-que desarrolla los principios de no-maleficencia, beneficencia, autonomía y justicia-, el médico deberá tratar de no hacer daño al paciente con su prescripción; que ésta le haga bien; respetar su autonomía y (de acuerdo con la formulación del principio de justicia distributiva) considerar que no vulnere la justa distribución de las cargas y los beneficios en la sociedad. Si bien era tópico en bioética afirmar que el médico en su consultorio no debería tener en cuenta cuestiones de justicia distributiva, esto ha sido cuestionado por Norman Daniels, quien afirma que, en realidad, el médico se mueve todo el tiempo con este telón de fondo. Dice Daniels que, teniendo el médico un grupo concreto de pacientes (y esto ocurre cada vez más, por decisión de ambas partes o de terceros), el tiempo, la atención y dedica- ción que dedica a alguno en especial no debe ir en detrimento de los demás(2). Creo que todos los que practicamos la asistencia actualmente podemos coincidir con este punto de vista.

Sin embargo, en las circunstancias actuales del ejercicio de la medicina, es difícil seguir los principios de Georgetown, cuanto más que, en realidad, la isla de la metáfora de Schweninger está cada vez más poblada por el tercero pagador (sea que éste se llame Estado, Obra social, Prepaga), el contador de la institución donde uno trabaja, el abogado del paciente, etc.

Pero, yendo a la ética de la prescripción médica en cuanto a su dimensión social, es decir, al impacto social de las recetas que todos los médicos extendemos día a día, quiero referirme especialmente a lo que ocurre hoy en Argentina (aunque pretenda extender luego mis observaciones al escenario de la globalización) haciendo para empezar dos preguntas:

1) ¿Nos damos cuenta de que ese impacto implica hoy casi la tercera parte del gasto en salud ${ }^{2}$ ?

2) Dado ese tremendo efecto presupuestario en nuestras escuálidas finanzas, ¿estamos convencidos realmente de que lo recetado es imprescindible?

En mi época de estudiante en la Universidad de Buenos Aires, allá por los años 60, había una especie de "axioma" que circulaba por la facultad: "doctor, no le cuide el bolsillo a su

Se ha hecho tradicional atribuirle esta imagen a este gran médico, aunque se piensa que, en realidad, pertenece a un ignoto discípulo suyo. Ver al respecto Laín Entralgo, Antropología Médica. Barcelona: Salvat;1984: 350.

Distintas fuentes estiman que el gasto en medicamentos en la Argentina significa entre el 25 y el $30 \%$ del gasto en salud. (Comparativamente, en EE.UU. 10\%, Alemania 12,8\%, Canadá 15,2\%. Velásquez G. Medicamentos: ¿derecho o mercancía? Le Monde Diplomatique, Ediciones ConoSur (el Dipló), julio 2003: 32-33. Germán Velásquez es el Coordinador del Programa de Acción para los Medicamentos de la OMS). 
paciente; cuídele la salud". Yo lo he adaptado a los tiempos que corren de la manera que sigue: "doctor, si quiere cuidarle la salud a su paciente, cuídele el bolsillo". Porque los cambios desde aquel entonces fueron siempre en el sentido de que, por un lado, nunca hubo menos dinero, más inequidad y más exclusión social en la Argentina y, por el otro, nunca hubo medicamentos tan caros. Entonces, no tener hoy en cuenta estos presupuestos es llanamente inmoral.

Profundizando en el proceso de pauperización, es evidente que la situación socioeconómica en Argentina ha evolucionado en los últimos treinta años, de modo tal que se han polarizado tanto la estructura productiva como la social. Por un lado, se consolidó un núcleo duro, aunque pequeño, de economía formal, para dar respuesta a las necesidades de la sociedad, y, por el otro, crecieron la informalidad y la pobreza, dando lugar a la expansión de la marginalidad social.

A partir de 1995 los indicadores muestran que mermó no sólo la cantidad, sino también la calidad del trabajo en Argentina, lo cual implicó la pérdida de bienestar y calidad de vida de las familias que se vieron afectadas en las prestaciones básicas: salud, educación, alimentación $\mathrm{y}$ vivienda. Se fue desde un Estado ineficiente a un Estado ausente, resultando este último un elemento central en la configuración de la situación de fragilidad social que se padece actualmente, en la que la pobreza y la desafiliación social son las facetas más visibles.

Los datos muestran que la población creció en cuatro millones de personas en una década, de las cuales el 50\% son individuos en condiciones de trabajar ${ }^{3}$. En este contexto, el desempleo pasó en el mismo período de 7,5 a 17,4\% $\mathrm{y}$, entre los ocupados asalariados, el trabajo en negro creció del 26 al 41\%, lo cual significa

"PEA", personas económicamente activas. que un elevado número de individuos, aun teniendo trabajo, no tienen jubilación ni cobertura social ${ }^{4}$. También, cabe señalar que el salario medio real, si se toma 1995 como año base, cayó en un $30 \%$, lo que demuestra que las posibilidades de vivir dignamente se restringieron para la mayoría de los argentinos. En 2001 el $52 \%$ de la población era pobre, midiendo su situación por los niveles de ingresos. En este universo, muchos son nuevos pobres por falta de trabajo, y no tienen las estrategias de supervivencia de quienes estructuralmente padecen condiciones de pobreza. De este modo la estructura social se ha ido modificando: se achicó la clase media, se concentraron los ingresos en un sector reducido de población capaz de acceder a todos los bienes y servicios, y se amplio la base de indigencia. El llamado "Índice de Inequidad", evaluado periódicamente por el INDEC ${ }^{5}$, que mide la brecha entre los ingresos del 10\% más pobre de la población y el 10\% más rico, era de $1 / 15$ en los años $90,1 / 30$ en 2000 y llegó a 1/50 a principios de 2004 .

Las más recientes informaciones oficiales son auspiciosas: en los últimos siete semestres se ha producido un crecimiento sostenido del Producto Interno Bruto (PIB); se recuperó el sector de manufacturas, que había registrado la mayor expulsión de mano de obra en los años noventa; se crearon más de un millón de puestos de trabajo genuinos; descendieron los índices de pobreza; se estabilizó la caída del salario y mejoró la inversión. Sin embargo, sólo volver al punto de partida es todo un desafío.

Es que la situación de vulnerabilidad social hace que esté aumentando el porcentaje de la población que no podrá salir de la pobreza aun cuando mejoren sus ingresos. Y esto, por lo que

El 55\% de la población no tiene cobertura médica, afirma la Asociación Civil de Actividades Médicas Integradas (ACAMI). La Capital, Mar del Plata 2/8/04, p. 18. Instituto Nacional de Estadística y Censos de la República Argentina. 
sostiene el premio Nobel en Economía Amartya $\mathrm{Sen}^{6}$, está en relación con su condición, ya que ser pobre es, entre otras cuestiones, la imposibilidad de dejar de serlo. En este escenario, la nueva cuestión social se revela como el gran reto que nos involucra y compromete.

Esbozada esta situación, quiero volver ahora a nuestro tema de la correcta prescripción, tratando de considerar, al menos en forma mínima, cada una de las aristas de este problema.

Empezaré con las siguientes preguntas: ¿actualizamos nuestros conocimientos de terapéutica? ¿Por qué medios? Entre los posibles tratamientos farmacológicos, ¿consideramos las opciones menos onerosas y las medidas nofarmacológicas? ¿Medicamos si realmente no hay otra opción, o es que muchas veces la receta reemplaza el diálogo franco que permita al paciente aceptar una situación inmodificable en vez de prescribir algo que sabemos que sólo actuará como placebo? ¿Nunca prescribimos para que el paciente no se vaya a buscar otro médico porque no le prescribimos nada (o aspirina), porque para eso no valía la pena que hubiera venido?

Esta batería de interrogantes nos concierne a los médicos casi en exclusividad, hace a la deontología profesional, y es básicamente técnica; se diría que la técnica correcta de la prescripción, según la lex artis. Teniendo como marco la moderna medicina basada en la evidencia(3-6), con sus revisiones sistemáticas y recomendaciones, además de las orientaciones aportadas por la OMS, podemos hacer un análisis al respecto.

La OMS mantiene desde 1978 una lista de medicamentos esenciales, periódicamente actualizada, que orienta sobre cuáles son los fár-

Sen Amartya. Public Action and the Quality of Life in Developing Countries. Oxford Bulletin of Economics and Statistics 1981; 43(4): 287-319. macos seguros y efectivos (como dicen, de la "experiencia" hemos pasado a la "evidencia"). Es bueno, quizás, recordar que esta lista incluye hoy sólo 320 medicamentos(7), en comparación con los miles que se comercializan en el mercado. Valen aquí varias aclaraciones: 1) "efectivos" se refiere a que tengan un buen perfil de eficacia en la práctica asistencial y, en realidad, se busca que sean costo/efectivos; 2) el listado pretende cubrir todas "las necesidades prioritarias de salud de la población"; y 3 ) la lista puede ser adaptada por cada región o país según sus patologías prevalentes.

Entre los libros y otras publicaciones referenciadas en el sitio web de la $\mathrm{OMS}^{7}$, en el prólogo de la "Guía de la buena prescripción", llamado "Por qué se necesita este libro", se dice: "...la formación clínica de pregrado se centra a menudo en las capacidades diagnósticas, más que en las terapéuticas (...) Los textos y formularios de referencia en farmacología suelen orientarse al medicamento y, aunque los libros de texto de materias clínicas y los protocolos terapéuticos están orientados a la enfermedad y dan recomendaciones terapéuticas, en ellos raramente se explica por qué se seleccionan estos tratamientos (...) Los malos hábitos de prescripción son el origen de tratamientos inefectivos e inseguros, de exacerbación o alargamiento de la enfermedad, de sufrimiento y daño al paciente y de costos más altos. También contribuyen a que quien prescribe sea vulnerable a influencias que pueden originar una prescripción irracional, como la presión del paciente, los malos ejemplos de los colegas y la información provista por los representantes de la industria(8)".

Ahora bien, demos por supuesto que, técnicamente, estamos en condiciones de prescribir correctamente. Cuando optamos por prescribir,

World Health Organization website: http://www.who.org 
nuestra decisión, de tanta gravitación como estoy tratando de mostrar, ¿es libre y responsable? ¿Qué hay de la manipulación que las empresas farmacéuticas hacen sobre la gente en general y sobre los médicos en particular? ¿De la fuerza de la publicidad mediática sobre la gente? ¿De la conducta del farmacéutico cuando el paciente va a comprar lo que le prescribimos, o cuando le pide algo directamente? ${ }^{8}$

Pareciera que en un mundo capitalista globalizado no se puede evitar que el desarrollo, producción y distribución de medicamentos sigan sujetos a la ética de los negocios. El economista Geoffrey Sachs, en un artículo crítico de la industria, concluye que, si bien la historia demuestra que las investigaciones financiadas por el Estado son buenas en las ciencias básicas, "el sector privado, cuyo fundamento es el lucro, es el que mejor desarrolla e introduce nuevos productos".

Es claro que la ética y los fines de las empresas farmacéuticas (y los de tecnología médica) son diferentes de los de la medicina. Para la industria este es un negocio como cualquier otro, y la manipulación para promocionar las ventas, como se hace con cualquier bien de consumo, está demostrada en todo nivel. La industria influye indebidamente sobre la medicina:

Manzini JL. Las relaciones peligrosas: la medicina y el complejo médico industrial. III Jornadas de Bioética del Consejo Superior del Colegio de Médicos de la Provincia de Buenos Aires, Bahía Blanca, 4/10/02, publicado en http:/ /www.aabioetica.org/Columna de opinion También: "Las relaciones peligrosas: los médicos y la industria". Reunión semanal de Medicina Interna, Hospital Privado de Comunidad de Mar del Plata, 16/05/03. Ver también Cañas M. Ensayos Clínicos y boletines independientes. Evidencia (Bs. As.) 2004; 7 (3):65-68, y Angell M. The Truth about the Drug Companies. The New York Review of Books 2004;51(12) accesible en http://www.nybooks.com/articles/ 17244

9 Sachs G. Las patentes y los pobres. La Nación (Buenos Aires) 07/05/01, p. 17.
- Como acción directa sobre los médicos por los visitadores y los obsequios de las empresas.

- Como principal protagonista en la investigación clínica, fijando la agenda y las condiciones.

- Por su influencia en la educación médica y en la confección de guías de buena práctica clínica.

- Por la infiltración de las agencias reguladoras como la FDA y, directamente, de los gobiernos.

- Manipulando a las asociaciones de consumidores de medicamentos, a los farmacéuticos y, al menos en nuestro país, a los simples empleados de farmacia ${ }^{10}$.

- A través de la propaganda mediática sobre el público, prescriptores y financistas, lo que deja en evidencia la falta de cumplimiento de sus deberes por parte de todos los funcionarios que debieran controlarla ${ }^{11}$.

10 Ante la falta de cumplimiento de las regulaciones vigentes, son los empleados "adiestrados" por los farmacéuticos, o los otros empleados más antiguos en el "ABC" de la dispensación de medicamentos, quienes terminan orientando al cliente hacia qué producto comprar, según el genérico prescripto por el médico. Esta política del Ministerio de Salud de la Nación, que obliga a los médicos a prescribir por el nombre genérico, se plasmó en la ley nacional 25.649 de 2002. Las diversas provincias y la Ciudad Autónoma de Buenos Aires han ido adhiriendo a través de leyes particulares. La Provincia de Buenos Aires, por la ley 12.895 de 2002. Estos empleados también prescriben, ante el pedido del cliente, un antigripal, antibióticos, sildenafil y hasta psicofármacos.

11 Consejo Federal de Radiodifusión de la República Argentina (CONFER), con poder policial sobre lo que se emite por radio y TV. La publicidad mediática está permitida en nuestro país sólo para medicamentos de venta libre que, dicho sea de paso, cada vez son más. En otros países, como Nueva Zelanda y EE.UU., se permite también para los medicamentos de venta bajo receta. Ver: Collier J. Iheanacho I. The Pharmaceutical industry as an informant (Medicines, society and industry I). Lancet 2002; 360: 1405-1409. 
Estaremos de acuerdo en que esto debe cambiar. Graham Dukes, del Instituto de Farmacoterapia de la Universidad de Oslo, señala: "La industria farmacéutica es responsable, por un lado ante sus accionistas y, por el otro, ante la sociedad en su conjunto. Estas dos obligaciones pueden, en principio, ser cumplidas a la vez (...) La industria ha demostrado ser suficientemente flexible como para realizar cambios si la sociedad persiste en sus reclamos(9)".

Pero quizás la pregunta más de fondo, sobre todo si estamos en el terreno de la bioética, sea: ¿en qué fundamentos éticos y jurídicos, si los hay, se pueden apoyar las organizaciones médicas intermedias, estatales y supranacionales, tipo OMS, para movilizarse en este sentido?

Un marco teórico fuerte, difícilmente rebatible, es la apelación al Derecho a la Salud. Este derecho, incluido desde fin del siglo XVIII en el derecho a la vida entre los derechos de primera generación, $\mathrm{y}$, como tal, liberal, individual, de no interferencia (que nadie atente contra mi salud), fue evolucionando y, ya a principios del siglo XX, se empezó a considerar como un derecho social, positivo, prestacional, exigible al Estado entre los de segunda generación. Últimamente, tanto por su pretensión de amparar a la sociedad como tal como por la fuerte carga de solidaridad que implica, se lo ubica entre los derechos de tercera generación(10-12) ${ }^{12}$ con el nombre de Derecho a la Atención de la Salud (en realidad sería mejor llamarlo Derecho a la Asistencia Sanitaria).

\footnotetext{
12 Los derechos de tercera generación amparan los llamados "intereses difusos" de la comunidad como tal, sin que exista una titularidad individual precisa. El derecho a la asistencia sanitaria acarrea un gasto social e implica decisiones difíciles respecto de la asignación de recursos y de las prioridades, en competencia con otros derechos, de acuerdo a la disponibilidad de esos recursos por parte del Estado, lo que, entre otras cosas, tiene que ver con lo que los ciudadanos están dispuestos a gastar en los diversos rubros.
}

Porque el medicamento que el médico prescribe es uno de los tratamientos que puede indicar, y sin tratamientos volveríamos a la Viena del siglo XIX, donde Skoda diagnosticaba lo que Rokitansky confirmaba en la autopsia ${ }^{13}$. Es decir, la medicina es, sobre todo, tratamiento y, hoy más que nunca, intento de devolver la salud o de evitar la progresión de la enfermedad y la muerte anticipada, o, al menos, la mitigación del sufrimiento(13). Y cuando la prescripción es farmacológica, eso se logra sólo con medicamentos eficaces, seguros, disponibles y accesibles para todos los seres humanos.

A este derecho apelan los jueces al resolver los amparos, que casi todos los días aparecen en los diarios, y ordenan que el tercero pagador provea, vuelva a proveer o no deje de proveer el medicamento, generalmente caro $^{14}$, que el paciente reclama.

La asistencia sanitaria como derecho, exigible en justicia, puede justificarse tanto desde la ética marxista como desde el socialismo democrático (origen del Estado de Bienestar o Estado Benefactor), el utilitarismo, como lo hacen Beauchamp y Childress(14), o la teoría de la justicia de Rawls, que aparece en EE.UU. contemporáneamente con la decadencia del Estado Benefactor ante la crisis económica de los 70. La teoría de Rawls puede verse como una reformulación del pensamiento social democrático(15) y, dada su influencia en la bioética contemporánea, diré algo acerca de ella.

Para el filósofo John Rawls, profesor de Harvard desde 1962 y fallecido en 2002, la jus-

\footnotetext{
${ }^{13}$ Acerca de la personalidad de Skoda, ver: Sakula A. Joseph Skoda 1805-1881: a centenary tribute to a pioneer of thoracic medicine. Thorax 1981; 36: 404-411. El gran internista Skoda y el patólogo Rokitansky, ambos fundadores de la Escuela de Viena, compartían un profundo "nihilismo terapéutico", quizás comprensible dada la ineficacia de la terapéutica de la época.

14 "Medicamentos catástrofe" los llaman los administradores.
} 
ticia es equidad, concepción que lo ubica entre el liberalismo y el utilitarismo extremos. En realidad, la suya es una teoría general de la justicia que apunta hacia una sociedad justa y que considera que los bienes sociales primarios deben ser distribuidos equitativamente; esta distribución no incluye la salud, porque ésta, según Rawls, es un bien natural, como la inteligencia y el vigor $(16)^{15}$.

Algunos bioeticistas formularon teorías de justicia sanitaria a partir de sus ideas, por distintos caminos. Mencionaré sólo a Norman Daniels y a Ronald Green. Daniels interpreta que el derecho a la asistencia sanitaria forma parte del principio de igualdad de oportunidades de la teoría de Rawls. La salud (y quizás también la educación) no es un bien social primario, sino un instrumento para la justa competencia por esos bienes sociales(17). Green, por su parte, incluye directamente a la salud entre los bienes sociales primarios, introduciendo en su concepto de salud consideraciones ecológicas y de compromiso con las generaciones futuras(18).

De todos modos, hablamos de la salud como un bien social y no como un bien de consumo, que es como, en cambio, se la concibe desde la ética de los negocios. Bien social significa lo que se le debe a todo ser humano por el sólo hecho de serlo. Por lo tanto, nos encontramos en el ámbito de las obligaciones y de la posibilidad de regulaciones y restricciones al libre accionar empresario.

Pero la ética sólo obliga moralmente. Para que la obligación pueda ser exigible, debe enfrentársele un derecho. Es decir, se debe plasmar la ética jurídicamente. Minima moralia, como la llamaba Theodor Adorno(19): las obli-

15 Rawls menciona como bienes sociales a la libertad, la igualdad de oportunidades, la renta, la riqueza y las bases sociales del respeto mutuo. gaciones morales, pocas pero de gran envergadura, que toda la sociedad se obliga a respetar.

En este sentido, todo el cuerpo doctrinal que ha ido cuajando bajo el nombre de Derecho Internacional de los Derechos Humanos coloca al Derecho a la Asistencia Sanitaria entre los derechos humanos fundamentales, personalísimos, derivado del derecho a la vida, ya desde la Declaración de la ONU de 1948. Ésta, en su Art. 25, inc.1, señala: "Toda persona tiene derecho a un nivel de vida adecuado que le asegure, así como a su familia, la salud y el bienestar, y, en especial, la alimentación, el vestido, la vivienda, la asistencia médica y los servicios sociales necesarios; tiene, asimismo, derecho a los seguros en caso de desempleo, enfermedad, invalidez, viudez, vejez u otros casos de pérdida de sus medios de subsistencia por circunstancias independientes de su volun$\operatorname{tad}^{16 "}$.

Es evidente el trasfondo del Estado Benefactor, propio de la filosofía política de esa época en Occidente, progresivamente desmantelado por razones presupuestarias en todas partes(20-23) pero, sobre todo, en los países latinoamericanos, que adoptaron o aceptaron las políticas neoliberales, particularmente influyentes en la última década, siguiendo las recomendaciones de los organismos internacionales de crédito (debido a su gran dependencia de ellos), pretendiendo copiar el pésimo modelo del Managed Care estadounidense(24,25).

De la Declaración Universal de Derechos Humanos, el Derecho a la Asistencia Sanitaria fue pasando a las diferentes convenciones y tratados sobre derechos humanos ${ }^{17}$. En lo que nos

16 Asamblea General de las Naciones Unidas. Declaración Universal de Derechos Humanos, 10/12/1948.

17 Las declaraciones internacionales son metas, ideales recomendables, pero no son vinculantes; en cambio los tratados, pactos y convenciones son de cumplimiento obligatorio para los países que los han suscrito. 
concierne más de cerca, está recogido en la Convención Americana de Derechos Humanos, también conocida como Pacto de San José de Costa Rica, de 1969, que Argentina ratificó por la ley 23.054 de 1984. Desde la reforma constitucional de 1994 tiene, por el Art. 75 inc. 22, jerarquía constitucional. Muchas de las constituciones provinciales reformadas subsecuentemente lo recogen de manera explícita. La nueva Constitución de la Provincia de Buenos Aires dice, en su art. 36: "(la Provincia) reconoce los siguientes derechos sociales (...) Inc. 8 (Derecho) a la salud. La Provincia garantiza a todos sus habitantes el acceso a la salud en los aspectos preventivos, asistenciales y terapéuticos; sostiene el hospital público y gratuito en general, con funciones de asistencia sanitaria, investigación y formación; promueve la educación para la salud; la rehabilitación y la reinserción de las personas tóxico-dependientes. El medicamento, por su condición de bien social, integra el derecho a la salud; la Provincia, a los fines de su seguridad, eficacia y disponibilidad asegura, en el ámbito de sus atribuciones, la participación de profesionales competentes en su proceso de producción y comercialización".

Germán Velásquez, coordinador del Programa de Acción para los Medicamentos de la OMS, expresa: "La promoción del derecho a la salud implica garantizar el derecho a beneficiarse con los adelantos tecnológicos y el reconocimiento del valor supremo de la dignidad humana, principios reconocidos en numerosos tratados internacionales y aceptados por la mayoría de los Estados. En general, tanto las reglas del comercio como las de la economía deben contribuir al bienestar de la sociedad. Nunca constituirse en obstáculo para que una parte importante de dicha sociedad se beneficie con la riqueza y la prosperidad que el comercio, en principio se presume, puede aportar. La salud percibida como derecho fundamental constituye un bien colectivo que las autoridades públicas y sus instituciones deben proteger de manera activa. No hacerlo es aceptar una sociedad enferma. (...) si se considera al medicamento como una simple mercancía, la salud nunca será otra cosa que una extensión del mercado allí donde las curas y los tratamientos sólo estarán al alcance de los que disponen de suficiente poder adquisitivo"18.

En la Argentina de hoy todas estas afirmaciones de Velásquez cobran, me parece, un tinte particularmente dramático o, si se quiere, patético, enrostrándonos nuestra crónica incapacidad para afrontarlas resueltamente como sociedad y buscarles una solución digna y equitativa.

Agradezco profundamente a Estela Lanari, Magíster en Ciencias Sociales (mención Economía), por sus aportes en cuanto a un diagnóstico de la situación en que se desenvuelven hoy, en nuestro país, estas interacciones que he pretendido desentrañar; y a Mirta Bajardi, abogada, por la revisión de los aspectos jurídicos del trabajo. Ambas son integrantes, como yo, del Comité de Ética del Hospital Privado de Mar del Plata.

\footnotetext{
Velásquez G. Medicamentos: ¿derecho o mercancía? Le Monde Diplomatique, Ediciones ConoSur (el Dipló), julio 2003: 32-33.
} 


\section{Referencias}

1. Beauchamp TL, Childress JF. Principles of Biomedical Ethics. New York: Oxford University Press; 1979.

2. Daniels N. Ethical Foundations of the Clinton Administration's Proposed Health Care System. JAMA 1994; 271: 1189-1196.

3. Evidence-Based Medicine Working Group. Evidence-Based Medicine: A New Approach to Teaching the Practice of Medicine. JAMA 1992; 268(17): 2420-2425.

4. Sackett DL, Rosenberg WMC, Gray JAM, Haynes RB, Richardson WS. Evidence based medicine: what it is and what it isn't. It's about integrating individual clinical expertise and the best external evidence. BMJ 1996; 312: 71-72.

5. Culpepper L, Gilbert TT. Evidence and ethics. The Lancet 1999; 353: 829-831.

6. González Aguilar P. Pediatría basada en la evidencia (PBE). Comentario editorial. Arch Argent Ped 2001; 99(4): 2902.

7. Laing R, Waning B, Gray A, Ford N, 't Hoen E. 25 years of the WHO essential medicines lists: progress and challenges. The Lancet 2003; 361: 1723-1729.

8. Organización Mundial de la Salud. Programa de Acción sobre Medicamentos Esenciales: Guía de la buena prescripción. Ginebra: OMS/DAP/94.11; 1998.

9. Graham Dukes MN. Medicines, society and industry IV. Accountability of the pharmaceutical industry. The Lancet 2002; 360: 1682-1684.

10. Ekmekdjian MÁ. Manual de la Constitución Argentina. Buenos Aires: De Palma; 1991: 72-74,

11. Kraut AJ. Los Derechos de los Pacientes. Buenos Aires: Abeledo Perrot; 1997: 212-220.

12. Daniels N. Why Justice is Good for our Health? En: Lolas Stepke F, Agar Corbinos L, (eds.) Interfaces between bioethics and the empirical social sciences. Publication Series-2002. Santiago de Chile: Regional Program on Bioethics, PAHO, WHO; 2002: 37-52.

13. Manzini JL. Los fines de la medicina. En: Bioética Paliativa. La Plata: Quirón; 1997: 34.

14. Drane J. Cuestiones de justicia en la prestación de servicios de salud. Boletín de la Oficina Sanitaria Panamericana 1990; 108(5-6): 586-598.

15. Gracia Guillén D. ¿Qué es un sistema justo de servicios de salud? Principios para la asignación de recursos escasos. Boletín de la Oficina Sanitaria Panamericana 1990; 108(5-6): 570-585.

16. Rawls J. A theory of Justice. Cambridge, Mass.: The Belknap Press of Harvard University Press; 1971.

17. Daniels N. Just Health Care. Cambridge, Mass.: Cambridge University Press; 1985.

18. Green R. M. Health Care and Justice in Contract Theory Perspective. En Veatch RM, Branson R, (eds.) Ethics and Health Police. Cambridge, Mass.: Ballinger Publ. Co; 1976.

19. Adorno TW. Minima Moralia. Reflexiones sobre la vida dañada. Madrid: Taurus; 1987. 
Ética de la prescripción médica en un país pauperizado - J. Manzini

20. Martínez-Pérez J. Medicina y Felicidad en el Siglo de las Luces. Algunas consideraciones sobre el origen del binomio Salud-Políticas de Bienestar. Quirón 1992; 23(3):54-61.

21. Porras Gallo MI. Salud y bienestar: un elemento teórico del discurso médico español. Quirón 1992; 23(4): 44-52.

22. González de Pablo A. El surgimiento y conformación del Estado de Bienestar. Quirón 1992; 23(4): 52-61.

23. Huertas R. Crisis económica y política neoliberal en el sector salud. Quirón 1993; 24(1): 14-19.

24. Stocker K, Waitzkin H, Iriart C. The Exportation of Managed Care to Latin America. NEJM 1999; 340:6

25. Pérez-Stable EJ. Managed Care Arrives in Latin America. NEJM 1999; 340:1110-1112. 